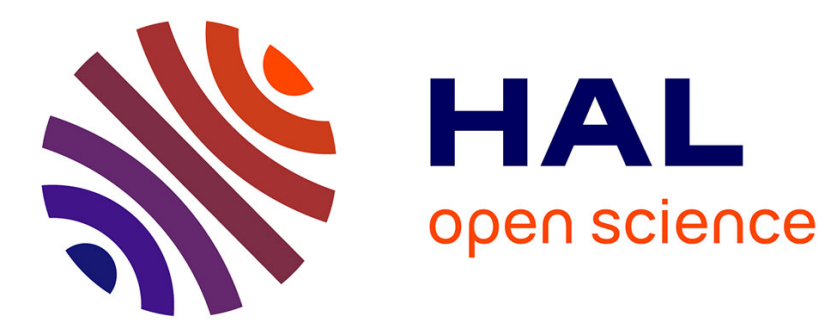

\title{
Instrumental Variable methods for closed-loop system identification
}

Marion Gilson, Paul van den Hof

\section{To cite this version:}

Marion Gilson, Paul van den Hof. Instrumental Variable methods for closed-loop system identification. Automatica, 2005, 41, pp.241-249. 10.1016/j.automatica.2004.09.016 . hal-00089684

\section{HAL Id: hal-00089684 https://hal.science/hal-00089684}

Submitted on 22 Aug 2006

HAL is a multi-disciplinary open access archive for the deposit and dissemination of scientific research documents, whether they are published or not. The documents may come from teaching and research institutions in France or abroad, or from public or private research centers.
L'archive ouverte pluridisciplinaire HAL, est destinée au dépôt et à la diffusion de documents scientifiques de niveau recherche, publiés ou non, émanant des établissements d'enseignement et de recherche français ou étrangers, des laboratoires publics ou privés. 


\title{
INSTRUMENTAL VARIABLE METHODS FOR CLOSED-LOOP SYSTEM IDENTIFICATION ${ }^{1}$
}

\author{
Marion Gilson ${ }^{*, 2}$ and Paul Van den Hof ${ }^{* *}$ \\ ${ }^{*}$ Centre de Recherche en Automatique de Nancy (CRAN), \\ CNRS UMR 7039, Université Henri Poincaré, Nancy 1, BP 239, \\ F-54506 Vandoeuvre-lès-Nancy Cedex, France. \\ Email: marion.gilson@cran.uhp-nancy.fr \\ ${ }^{* *}$ Delft Center for Systems and Control, Delft University of Technology, \\ Mekelweg 2, 2628 CD Delft, The Netherlands. \\ Email: p.m.j.vandenhof@dcsc.tudelft.nl
}

\begin{abstract}
In this paper, several instrumental variable (IV) and instrumental variable-related methods for closed-loop system identification are considered and set in an extended IV framework. Extended IV methods require the appropriate choice of particular design variables, as the number and type of instrumental signals, data prefiltering and the choice of an appropriate norm of the extended IV-criterion. The optimal IV estimator achieves minimum variance, but requires the exact knowledge of the noise model. For the closed-loop situation several IV methods are put in an extended IV framework and characterized by different choices of design variables. Their variance properties are considered and illustrated with a simulation example.
\end{abstract}

Keywords: Closed-loop system identification; linear estimators; instrumental variables.

\section{INTRODUCTION}

For many industrial production processes, safety and production restrictions are often strong reasons for not allowing identification experiments in open-loop. In such situations, experimental data can only be obtained under so-called closed-loop conditions. The main difficulty in closed-loop identification is due to the correlation between the disturbances and the control signal, induced by the loop. Several classical alternatives are available to cope with this problem, broadly classified into three main approaches: direct, indirect and joint input/output (Söderström and Stoica 1989, Ljung 1999). Some particular versions of these methods have been developed more recently in the area of control-relevant identification as e.g. the

\footnotetext{
1 Paper submitted for publication in Automatica. Submitted 30 January 2004; revised 12 July 2004.

2 Author to whom correspondence should be addressed, tel. +33 383684 475, fax: +33 383684462 .
}

two-stage, the coprime factor, the dual-Youla methods. An overview of these recent developments can be found in Van den Hof (1998) and Forssell and Ljung (1999).

When looking at methods that can consistently identify plant models of systems operating in closedloop while relying on simple linear (regression) algorithms, instrumental variable (IV) techniques seem to be rather attractive, but at the same time also not very often applied. On the other hand, when dealing with highly complex processes that are high dimensional in terms of inputs and outputs, it can be attractive to rely on methods that do not require non-convex optimization algorithms. Besides this computationally attractive property, IV methods have the potential advantage that they can identify plant models consistently also when the noise model is misspecified, and when the present controller is non-linear and/or time-varying. For closed-loop identification a basic IV estimator has been proposed (Söderström et al. 1987), and more 
recently a so-called tailor-made IV algorithm (Gilson and Van den Hof 2001), where the closed-loop plant is parametrized using (open-loop) plant parameters. The class of algorithms denoted by BELS (for BiasEliminated Least-Squares), e.g. Zheng (1996), is also directed towards the use of linear regression algorithms only. It has recently been shown that these algorithms are also particular forms of IV estimation schemes (Söderström et al. 1999, Gilson and Van den Hof 2001). Then, when comparing the several available IV algorithms, the principal question to address should be: how to achieve the smallest variance of the estimate. Concerning extended IV methods an optimal variance result has been developed in the openloop identification case, showing consequences for the choice of weights, filters, and instruments (Stoica and Söderström 1983, Söderström and Stoica 1989, Ljung 1999). For the closed-loop case a covariance analysis has been provided by Söderström et al. (1987) and Söderström and Stoica (1983). While in Forssell and Chou (1998) this analysis is used to compare several closed-loop identification methods, in the present paper main attention will be given to a characterization of the properties of the several (extended) IV-methods presented here.

The paper is organized as follows. After the preliminaries, several IV and IV-related methods are presented and unified in an extended IV framework in section 3. Section 4 introduces the optimum variance closed-loop IV estimation with the consequences for the several design variables. Since for optimum variance, the noise model has to be known exactly, several bootstrap methods are proposed in section 5 for approximating this required information from measurement data. In section 6 , the comparison between the different proposed methods is illustrated in a simulation example, showing that the optimal estimator can be accurately approximated by an appropriate choice of the design parameters.

\section{PRELIMINARIES}

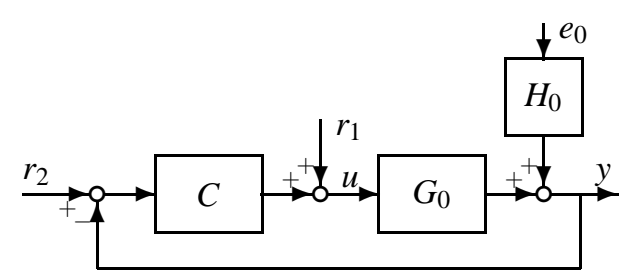

Fig. 1. Closed-loop configuration.

Consider a linear SISO closed-loop system shown in figure 1 . The process is denoted by $G_{0}(z)$ and the controller by $C(z) ; u(t)$ describes the process input signal, $y(t)$ the process output signal and $\left\{e_{0}(t)\right\}$ is a sequence of independent identically disturbed random variables of variance $\lambda_{0}$. The external signals $r_{1}(t)$, $r_{2}(t)$ are assumed to be uncorrelated with $e_{0}(t)$. For ease of notation we also introduce the signal $r(t)=$
$r_{1}(t)+C(q) r_{2}(t)$. With this notation, the data generating system becomes

$$
\mathcal{S}:\left\{\begin{array}{l}
y(t)=G_{0}(q) u(t)+H_{0}(q) e_{0}(t) \\
u(t)=r(t)-C(q) y(t)
\end{array}\right.
$$

The real plant $G_{0}$ is considered to satisfy $G_{0}(q)=$ $B_{0}\left(q^{-1}\right) / A_{0}\left(q^{-1}\right)$, while in these expressions $q^{-1}$ is the delay operator, and the numerator and denominator degree is $n_{0}$. The $m$-th order controller $C$ is assumed to be known and specified by

$$
C(q)=\frac{Q\left(q^{-1}\right)}{P\left(q^{-1}\right)}=\frac{q_{0}+q_{1} q^{-1}+\cdots+q_{m} q^{-m}}{1+p_{1} q^{-1}+\cdots+p_{m} q^{-m}}
$$

with the pair of polynomials $(P, Q)$ assumed to be coprime.

A parametrized process model is considered

$$
\mathcal{G}: G(q, \theta)=\frac{B\left(q^{-1}, \theta\right)}{A\left(q^{-1}, \theta\right)}=\frac{b_{1} q^{-1}+\cdots+b_{n} q^{-n}}{1+a_{1} q^{-1}+\cdots+a_{n} q^{-n}},
$$

and the process model parameters are stacked columnwise in the parameter vector

$$
\boldsymbol{\theta}=\left[\begin{array}{llllll}
a_{1} & \cdots & a_{n} & b_{1} & \cdots & b_{n}
\end{array}\right]^{T} \in \mathbb{R}^{2 n} .
$$

Furthermore, let us denote by $\varphi_{c}(t)$ and by $\varphi(t)$ the closed-loop and open-loop regressors respectively, defined as

$$
\begin{aligned}
\varphi_{c}^{T}(t)= & {[-y(t-1) \cdots-y(t-n-m)} \\
& r(t-1) \cdots r(t-n-m)] \in \mathbb{R}^{2 n+2 m} \\
\varphi^{T}(t)= & {[-y(t-1) \cdots-y(t-n)} \\
& u(t-1) \cdots u(t-n)] \in \mathbb{R}^{2 n} \\
\varphi_{r}^{T}(t)= & {\left[r(t-1) \cdots r\left(t-r_{B}\right)\right] \in \mathbb{R}^{r_{B}} . }
\end{aligned}
$$

and $r_{B}$ a user-specified integer. If $n=n_{0}$, i.e. the plant $G_{0}$ is contained in the chosen model set, the output $y(t)$ can be written as

$$
y(t)=\varphi^{T}(t) \theta_{0}+v_{0}(t)
$$

where $\theta_{0}$ denotes the true parameters and $v_{0}(t)=$ $A_{0}\left(q^{-1}\right) H_{0}(q) e_{0}(t)$. Additionally we use the following notation for filtered data

$$
\begin{aligned}
\bar{\varphi}(t) & =L\left(q^{-1}\right) \varphi(t) \\
\bar{y}(t) & =L\left(q^{-1}\right) y(t)
\end{aligned}
$$

where $L\left(q^{-1}\right)$ is a particular chosen prefilter.

\section{CLOSED-LOOP IV METHODS IN AN EXTENDED IV FRAMEWORK}

\subsection{Closed-loop basic IV method}

The basic-IV estimate of $\theta_{0}$ is determined as the solution $\hat{\theta}_{i v}$ to the set of equations

$$
\frac{1}{N} \sum_{t=1}^{N} z(t)\left[y(t)-\varphi^{T}(t) \hat{\theta}_{i v}\right]=0
$$


or, provided that $\sum_{t=1}^{N} z(t) \varphi^{T}(t)$ is non singular

$$
\hat{\theta}_{i v}=\left[\frac{1}{N} \sum_{t=1}^{N} z(t) \varphi^{T}(t)\right]^{-1}\left[\frac{1}{N} \sum_{t=1}^{N} z(t) y(t)\right]
$$

where $z(t)$ represents the vector of instruments, having dimension $2 n$.

By using equation (7) in (11), it follows that

$$
\hat{\theta}_{i v}=\theta_{0}+\left[\frac{1}{N} \sum_{i=1}^{N} z(t) \varphi^{T}(t)\right]^{-1}\left[\frac{1}{N} \sum_{i=1}^{N} z(t) v_{0}(t)\right]
$$

Therefore, the basic IV estimate provides a consistent parameter estimate $\left(\operatorname{plim}_{N \rightarrow \infty} \hat{\theta}=\theta_{0}\right)$ under the following two conditions ${ }^{3}$ :

- $\overline{\mathbb{E}} z(t) \varphi^{T}(t)$ is non singular,

- $\overline{\mathbb{E}} z(t) v_{0}(t)=0$.

A typical choice in the closed-loop situation is that the instruments vector is composed of delayed samples of the reference signel: $z(t)=\varphi_{r}(t)$ with dimension $r_{B}=2 n$. Note that the consistency result is valid under the condition that $G_{0}$ is contained in the chosen model set; it does not require exact modeling of the noise model $H_{0}$ and it allows the controller $C$ to be nonlinear and or time-varying.

\subsection{Closed-loop extended IV method}

An extended IV estimate of $\theta_{0}$ is obtained by generalizing the so-called basic IV estimates of $\theta$ by prefiltering the data and by using an augmented instrument $z(t) \in \mathbb{R}^{n_{z}}\left(n_{z}>2 n\right)$ so that (10) leads to an overdetermined set of equations that is solved by:

$$
\begin{aligned}
\hat{\theta}_{x i v}(N)=\arg \min _{\theta} & \|\left[\frac{1}{N} \sum_{t=1}^{N} z(t) L(q) \varphi^{T}(t)\right] \theta \\
& -\left[\frac{1}{N} \sum_{t=1}^{N} z(t) L(q) y(t)\right] \|_{Q}^{2},
\end{aligned}
$$

where $L(q)$ is a stable prefilter and $\|x\|_{Q}^{2}=x^{T} Q x$, with $Q$ a positive definite weighting matrix.

Following the same reasoning as in the basic IV case, the extended-IV estimate provides a consistent estimate under the following two conditions

- $\mathbb{E} z(t) L(q) \varphi^{T}(t)$ is non singular,

- $\overline{\mathbb{E}} z(t) L(q) v_{0}(t)=0$.

\subsection{Tailor-made IV identification (M1)}

The tailor-made IV method (referred to M1 in the following) as discussed in (Gilson and Van den Hof 2001) is designed to provide an unbiased estimate for

3 The notation $\overline{\mathbb{E}}[]=.\lim _{N \rightarrow \infty} \frac{1}{N} \sum_{t=0}^{N-1} \mathbb{E}[$.$] is adopted from the$ prediction error framework of Ljung (1999) the process model $G(q, \theta)$, while pertaining to simple linear regression type of estimates. The closed-loop transfer function from $r$ to $y$ is modelled by

$\frac{G(q, \theta)}{1+C(q) G(q, \theta)}=\frac{B\left(q^{-1}, \theta\right) P\left(q^{-1}\right)}{A\left(q^{-1}, \theta\right) P\left(q^{-1}\right)+B\left(q^{-1}, \theta\right) Q\left(q^{-1}\right)}$,

parametrized in the plant parameter $\theta$ (tailor-made parametrization). The prediction error related to a linear regression model for the closed-loop system is then given by

$$
\begin{aligned}
& \varepsilon(t, \theta)=\bar{A}_{c l}\left(q^{-1}, \theta\right) y(t)-\bar{B}_{c l}\left(q^{-1}, \theta\right) r(t) \\
& \bar{B}_{c l}\left(q^{-1}, \theta\right)=B\left(q^{-1}, \theta\right) P\left(q^{-1}\right) \\
& \bar{A}_{c l}\left(q^{-1}, \theta\right)=A\left(q^{-1}, \theta\right) P\left(q^{-1}\right)+B\left(q^{-1}, \theta\right) Q\left(q^{-1}\right) .
\end{aligned}
$$

Using the relation $r=u+C y$ or equivalently $P r=$ $P u+Q y$, it follows that

$\varepsilon(t, \theta)=A\left(q^{-1}, \theta\right) P\left(q^{-1}\right) y(t)-B\left(q^{-1}, \theta\right) P\left(q^{-1}\right) u(t)$,

which alternatively can be written as

$$
\varepsilon(t, \theta)=\bar{y}(t)-\bar{\varphi}^{T}(t) \theta .
$$

with $\bar{y}(t), \bar{\varphi}^{T}(t)$ given by (8)-(9), and the prefilter $L(q)$ particularly chosen as $L(q)=P\left(q^{-1}\right)$. Then the tailormade IV estimate of $\theta$ is determined as the solution to the set of equations

$$
\frac{1}{N} \sum_{t=1}^{N} \varepsilon\left(t, \hat{\theta}_{t i v, F}\right) \eta(t)=0
$$

where $\eta(t)=F \varphi_{r}(t)$ denotes the vector of instruments, and $F \in \mathbb{R}^{2 n \times r_{B}}$ a user-chosen matrix with rank $2 n$.

The choice $r_{B}=2 n, F=I_{2 n}$ leads to a simple basic IV estimator applied to the closed-loop system, taking as instruments $2 n$ delayed samples of the reference signal, that is supposed to be persistently exciting of sufficiently high order. For $r_{B}>2 n$, the matrix $F$ constructs $2 n$ instruments out of $r_{B}$ delayed reference samples, by taking particular linear combinations. Again a basic IV method results, but now with a particular choice of instruments.

\subsection{BELS method}

The so-called bias-eliminated least-squares method (BELS) as proposed by (Zheng and Feng 1995, Zheng 1996) has been shown to be a particular form of tailormade IV estimator (Gilson and Van den Hof 2001). It has two different formats, dependent on the relation between $n$ (model order) and $m$ (controller order). For $m \leq n$ the BELS estimator is equivalent to the tailormade estimate with $r_{B}=2 n$ and $F=I_{2 n}$. For $m>n$ it is obtained by choosing $r_{B}=n+m$ and

$$
F=M^{T} \hat{R}_{\varphi_{r} \varphi_{c}}^{T}(N)\left(\hat{R}_{\varphi_{r} \varphi_{c}}(N) \hat{R}_{\varphi_{r} \varphi_{c}}^{T}(N)\right)^{-1} .
$$

with $\quad \hat{R}_{\varphi_{r} \varphi_{c}}(N)=\frac{1}{N} \sum_{t=1}^{N} \varphi_{r}(t) \varphi_{c}^{T}(t), \quad$ and $M \in \mathbb{R}^{\left(n+m+r_{B}\right) \times 2 n}$ a full-column rank matrix 
dependent on controller dynamics only and specified as

$$
M=\left(\begin{array}{cc}
P_{c} & Q_{c} \\
0 & \bar{P}_{c}
\end{array}\right) \in \mathbb{R}^{\left(n+m+r_{B}\right) \times 2 n}
$$

where $P_{c}, Q_{c} \in \mathbb{R}^{(n+m) \times n}$ are Sylvester matrices expanded by $\left[\begin{array}{llll}1 & p_{1} & \cdots & p_{m}\end{array}\right]^{T}$ and $\left[\begin{array}{llll}q_{0} & q_{1} & \cdots & q_{m}\end{array}\right]^{T}$ respectively, given in (2), i.e.

$$
P_{c}=\left[\begin{array}{cccc}
1 & 0 & \cdots & 0 \\
p_{1} & \ddots & \ddots & \vdots \\
\vdots & \ddots & \ddots & 0 \\
p_{m} & \vdots & \ddots & 1 \\
0 & \ddots & \vdots & p_{1} \\
\vdots & \ddots & \ddots & \vdots \\
0 & \cdots & 0 & p_{m}
\end{array}\right]
$$

and $\bar{P}_{c} \in \mathbb{R}^{r_{B} \times n}$ is given by $P_{c}$ but expanded with a zero matrix:

$$
\bar{P}_{c}=\left[\begin{array}{c}
P_{c} \\
0_{\left(r_{B}-n-m\right) \times n}
\end{array}\right] .
$$

The matrix $M$ satisfies

$$
\bar{\varphi}(t)=M^{T} \varphi_{c}(t) ;
$$

for a full description of the relation between BELS and tailor-made IV, see Gilson and Van den Hof (2001).

\subsection{Tailor-made and extended IV identification}

3.5.1. Unification In order to analyse the variance properties of the estimators presented above, they are positioned in the framework of extended IV estimators. All estimators discussed so far can simply be interpreted as a special form of extended IV estimator. The most complicated one is the BELS estimator specified by the particular choice of instruments determined by $F$ in (17) and referring to the situation $m>n$ only. For this latter estimator an alternative formulation can be given, as shown in the next Proposition.

Proposition 1. The tailor-made IV estimates presented in sections 3.3 and 3.4 related to the particular situation $m>n, r_{B}=n+m$ and with the particular choice of $F$ given in (17), satisfies

$$
\hat{\theta}_{t i v}(N)=\arg \min _{\theta}\left\|\hat{R}_{\varphi_{r} \bar{\varphi}}(N) \theta-\hat{R}_{\varphi_{r}{ }^{-}}(N)\right\|_{Q}^{2}
$$

with $\quad \hat{R}_{\varphi_{r} \bar{\varphi}}(N)=\frac{1}{N} \sum_{t=1}^{N} \varphi_{r}(t) \bar{\varphi}^{T}(t), \quad \hat{R}_{\varphi_{r}-\bar{y}}(N)=$ $\frac{1}{N} \sum_{t=1}^{N} \varphi_{r}(t) \bar{y}(t)$, and

$$
Q=\left(\hat{R}_{\varphi_{r} \varphi_{c}} \hat{R}_{\varphi_{r} \varphi_{c}}^{T}\right)^{-1} \in \mathbb{R}^{(n+m) \times(n+m)} .
$$

Consequently it is equivalent to an extended IV estimator (13) with

- instrument vector $z(t)=\varphi_{r}(t)$ with dimension $n_{z}=r_{B}=n+m$

- prefilter $L(q)=P\left(q^{-1}\right)$,

- weighting matrix $Q(22)$.
Proof. A full proof is added in the appendix.

As a result, all considered IV methods can be unified in an extended IV framework, where the estimator is specified by equation (13) with particular choices of the design parameters $z(t), L(q)$ and $Q$, as summarized in the following overview:

- closed-loop basic IV (section 3.1): $n_{z}=2 n$, $z(t)=\varphi_{r}(t)$ with $r_{B}=2 n, L=1, Q=I$,

- tailor-made IV (section 3.3): $n_{z}=2 n, z(t)=$ $F(q) \varphi_{r}(t)$ with $r_{B}=2 n, L=P\left(q^{-1}\right), Q=I$,

- BELS in case $m \leq n$ (section 3.4): special case of the tailor-made IV where $F=I$ and $r_{B}=2 n$,

- BELS in case $m>n$ (see proposition 1): $n_{z}=n+$ $m, z(t)=\varphi_{r}(t)$ with $r_{B}=n+m, L=P\left(q^{-1}\right)$, $Q=\left(\hat{R}_{\varphi_{r} \varphi_{c}} \hat{R}_{\varphi_{r} \varphi_{c}}^{T}\right)^{-1}$.

3.5.2. Covariance property The asymptotic distribution of the parameter (13) estimated by an extended IV type of method has been extensively investigated in the open-loop context (Söderström and Stoica 1989). Moreover, the structure of the closed-loop extended IV estimates is identical to that of the open-loop estimates, as is shown in (Söderström et al. 1987). As a result, under the assumptions given in section 3.2 (in particular that instruments and noise are uncorrelated) and $G_{0} \in \mathcal{G}, \hat{\theta}$ is asymptotically Gaussian distributed

$$
\sqrt{N}\left(\hat{\theta}-\theta^{*}\right) \stackrel{\text { dist }}{\rightarrow} \mathcal{N}\left(0, P_{\text {xiv }}\right)
$$

with $\theta^{*}$ the asymptotic parameter estimate, and the covariance matrix $P_{x i v}$ is given by

$$
P_{x i v}=\lambda_{0}\left(R_{z \bar{\varphi}}^{T} Q R_{z \bar{\varphi}}\right)^{-1} R_{z \bar{\varphi}}^{T} Q R_{z_{T} z T} Q R_{z \bar{\varphi}}\left(R_{z \bar{\varphi}}^{T} Q R_{z \bar{\varphi}}\right)^{-1}
$$

where

$$
\begin{aligned}
R_{z \bar{\varphi}} & =\overline{\mathbb{E}} z(t) \bar{\varphi}^{T}(t)=\overline{\mathbb{E}} z(t) L(q) \varphi^{T}(t) \\
R_{z_{T} z_{T}} & =\overline{\mathbb{E}} z_{T}(t) z_{T}^{T}(t) \\
z_{T}(t) & =\sum_{i=0}^{\infty} t_{i} z(t+i) \\
T(q) & =L(q) A_{0}\left(q^{-1}\right) H_{0}(q)=\sum_{i=0}^{\infty} t_{i} q^{-i}
\end{aligned}
$$

and $T(q)$ is a monic filter.

3.5.3. Remarks In the situation $n_{z}=2 n$, the number of unknowns in (13) is equal to the number of equation, and the weighting matrix $Q$ will not influence the optimization. In that situation the simple choice $Q=I$ suffices. According to equation (24) and under the assumption $G_{0} \in \mathcal{G}$, the expression for the covariance matrix of this estimate then simplifies to

$$
P_{x i v}=\lambda_{0} R_{z \bar{\varphi}}^{-1} R_{z_{T} z_{T}} R_{z \bar{\varphi}}^{-T}
$$

\section{OPTIMAL CLOSED-LOOP IV}

The choice of the instruments $z(t)$, of $n_{z}$, of the weighting matrix $Q$ and of the prefilter $L(q)$ may have 
a considerable effect on the covariance matrix $P_{x i v}$. In the open-loop situation the lower bound of $P_{x i v}$ for any unbiased identification method is given by the Cramer-Rao bound, which is specified in e.g. Ljung (1999) and Söderström and Stoica (1983). Optimal choices of the above mentioned design variables exist so that $P_{x i v}$ reaches the Cramer Rao bound. For the closed-loop case, this type of reasoning is not viable for IV estimates, as the objective of reaching minimum variance conflicts with the restriction that instruments and noise should be uncorrelated. However it has been shown in (Söderström et al. 1987) that there indeed exists a minimum value of the covariance matrix $P_{x i v}$ as a function of the design variables $z(t)$, $L(q)$ and $Q$, under the restriction that $z(t)$ is a function of the external signal $r(t)$ only:

$$
P_{x i v} \geq P_{x i v}^{o p t}
$$

with

$$
P_{x i v}^{o p t}=\lambda_{0}\left[\overline{\mathbb{E}} \tilde{\varphi}_{F}(t) \tilde{\varphi}_{F}^{T}(t)\right]^{-1},
$$

$\tilde{\varphi}_{F}(t)=L(q) \tilde{\varphi}(t)$ and $\tilde{\varphi}(t)$ is the noise-free part of $\varphi(t)$.

The minimum variance can be achieved by the following choice of design variables:

- $Q=I$ and $n_{z}=2 n$, i.e. $R_{z \bar{\varphi}}$ in (24) is square;

- $L(q)=\frac{1}{H_{0}(q) A_{0}\left(q^{-1}\right)}$

- $z(t)=\frac{1}{H_{0}(q) A_{0}\left(q^{-1}\right)} \tilde{\varphi}(t)$

This can be verified by subsitution into (24) and by using the fact that

$$
R_{z \bar{\varphi}}=\overline{\mathbb{E}} z(t) L(q) \tilde{\varphi}^{T}(t)=\overline{\mathbb{E}} \tilde{\varphi}_{F}(t) \tilde{\varphi}_{F}^{T}(t) .
$$

Note that the optimal IV estimator can only be obtained if the true noise model $A_{0}\left(q^{-1}\right) H_{0}(q)^{4}$ is exactly known and therefore optimal accuracy cannot be achieved in practice.

Furthermore, it is interesting to notice that the optimal accuracy is achieved without introducing any additional instruments $\left(n_{z}=2 n\right)$, like in the open-loop situation. The introduction of additional instruments will not contribute to reduction of the variance, at least in the ideal situation of an exactly known noise model $A_{0}\left(q^{-1}\right) H_{0}(q)$.

\section{APPROXIMATE IMPLEMENTATIONS}

In order to give some clues to the closed-loop identification method users, it would be interesting to compare the extended IV method with the optimal IV one. However, as the latter cannot be achieved in practice, approximate implementations of the optimal IV method will be considered. For this purpose one will need to take care that

- a model of $A_{0} H_{0}$ is available in order to construct the prefilter $L(q)$ and the instruments $z(t)$,

4 Although $A_{0} H_{0}$ is referred to as "noise model" it also involves knowledge of $G_{0}$ through its denominator $A_{0}$.
- a first model of $G_{0}(q)$ is needed to compute the noise free part of the regressor $\tilde{\varphi}(t)$.

The choice of the instruments and prefilter in the IV method affects the asymptotic variance, while consistency properties are generically secured. This suggests that minor deviations from the optimal value (which is not available in practice) will only cause secondorder effects in the resulting accuracy. Therefore it is considered to be sufficient to use consistent, but not necessarily efficient estimates of the dynamics and of the noise when constituting the instrument and the prefilter (Ljung 1999).

Additionally for obtaining the necessary preliminary models a restriction is made to linear regression estimates in order to keep computational procedures simple and tractable.

\subsection{First alternative (M2, M3)}

Several bootstrap IV methods have been proposed in the open-loop situation, in an attempt to approximate the optimal IV method, see e.g. (Young 1976, Söderström and Stoica 1983, Ljung 1999). A first solution consists in extending one of these algorithms to the closed-loop situation; here the IV4 method (Ljung 1999) will be considered. The only difference between open-loop and closed-loop cases is that in the latter, also the input is correlated with the noise. Therefore, the instruments have to be uncorrelated with the noise part of $u(t)$ but correlated with the noise-free part of $u(t)$.

\section{Method M2 (cliv4).}

Step 1. Write the model structure as a linear regression

$$
\hat{y}(t, \theta)=\varphi(t)^{T} \theta \text {. }
$$

Estimate $\theta$ by a least-squares method and get $\hat{\theta}_{1}$ along with the corresponding transfer function $\hat{G}_{1}(q)$.

Step2. Generate the instruments $z_{1}(t)$ as

$$
\begin{aligned}
\tilde{y}_{1}(t) & =\frac{C(q) \hat{G}_{1}(q)}{1+C(q) \hat{G}_{1}(q)} r(t) \\
\tilde{u}_{1}(t) & =\frac{1}{1+C(q) \hat{G}_{1}(q)} r(t) \\
z_{1}(t) & =\left[-\tilde{y}_{1}(t-1) \cdots-\tilde{y}_{1}(t-n) \tilde{u}_{1}(t-1) \cdots \tilde{u}_{1}(t-n)\right]^{T}
\end{aligned}
$$

$z_{1}(t)$ can be seen as an estimation of the noisefree part of the regressor $\varphi(t)$. Determine the IV estimate of $\theta$ in (30) as

$$
\hat{\theta}_{2}=\hat{R}_{z_{1} \varphi}^{-1} \hat{R}_{z_{1} y}
$$

The corresponding estimated transfer function is given by $\hat{G}_{2}(q)=\frac{\hat{B}_{2}\left(q^{-1}\right)}{\hat{A}_{2}\left(q^{-1}\right)}$, of order $n$.

Step 3. Let $\hat{w}(t)=\hat{A}_{2}\left(q^{-1}\right) y(t)-\hat{B}_{2}\left(q^{-1}\right) u(t)$ and postulate an AR model of order $2 n$ for $\hat{w}(t)$ : $L(q) \hat{w}(t)=e(t)$.

Estimate $L(q)$ using a least-squares method and denote the result by $\hat{L}(q)$. 
Step 4. Generate the instruments $z_{2}(t)$ as

$$
\begin{aligned}
\tilde{y}_{2}(t) & =\frac{C(q) \hat{G}_{2}(q)}{1+C(q) \hat{G}_{2}(q)} r(t) \\
\tilde{u}_{2}(t) & =\frac{1}{1+C(q) \hat{G}_{2}(q)} r(t) \\
z_{2}(t) & =\left[-\tilde{y}_{2}(t-1) \cdots-\tilde{y}_{2}(t-n) \tilde{u}_{2}(t-1) \cdots \tilde{u}_{2}(t-n)\right]^{T}
\end{aligned}
$$

Using these instruments $z_{2}(t)$ and the prefilter $\hat{L}(q)$, determine the IV estimate of $\theta$ in (30) as

$$
\hat{\theta}_{c l i v 4}=\hat{R}_{z_{2} \varphi_{T}}^{-1} \hat{R}_{z_{2} y_{T}},
$$

where

$$
\varphi_{T}(t)=\hat{L}(q) \varphi(t) \text { and } y_{T}(t)=\hat{L}(q) y(t) .
$$

The asymptotic covariance matrix of the final estimates is the Cramer-Rao bound, provided the true noise model is an autoregression of order $2 n$.

Method M3 (cliv4-armasel). The method above can be improved by using a more sophisticated noise modeling procedure, e.g. by replacing the third step of the M2 algorithm by the armasel procedure developed in Broersen (2002), including an appropriate order selection step. This procedure consists in estimating several autoregressive models of different orders and in applying a nonasymptotic order selection criterion based on estimates of prediction error expectation.

\subsection{Second alternative (M4)}

Noise and process models have to be known in order to construct the instruments and the prefilter. Since, the second order statistical property is not of crucial importance, a simple solution consists in estimating these models by using a high-order least-squares estimator. The result will be obviously biased but a bias in the first step does not lead to a bias in the final model.

\section{Method M4 (cliv3).}

Step 1. Write the model structure as a linear regression (30), and estimate $\theta$ by a high-order leastsquares method.

The resulting $\hat{\theta}_{1}$ leads to process and noise models $\hat{G}_{1}(q)=\frac{\hat{B}_{1}\left(q^{-1}\right)}{\hat{A}_{1}\left(q^{-1}\right)}, \hat{H}_{1}(q)=\frac{1}{\hat{A}_{1}\left(q^{-1}\right)}$ respectively.

Step 2. The prefilter $\hat{L}(q)=\hat{A}_{1}\left(q^{-1}\right) \hat{H}_{1}(q)=1$ by construction, due to the ARX model structure. Compute the noise-free part of the regressor

$$
\tilde{\varphi}(t)=\left[-\tilde{y}_{1}(t-1) \cdots-\tilde{y}_{1}(t-n) \tilde{u}_{1}(t-1) \cdots \tilde{u}_{1}(t-n)\right]^{T}
$$

with $\tilde{y}_{1}(t)$ and $\tilde{u}_{1}(t)$ computed as in equations (31)(32). Generate the instruments as

$$
z(t)=\left\{\left[\hat{A}_{1}\left(q^{-1}\right) \hat{H}_{1}(q)\right]^{-1} \tilde{\varphi}^{T}(t)\right\}^{T}
$$

Step 3. Using the instrument $z(t)$ and the prefilter $\hat{L}(q)$, determine the IV estimate in (30) as

$$
\hat{\theta}_{\text {cliv } 3}=\hat{R}_{z \varphi_{T}}^{-1} \hat{R}_{z y_{T}} .
$$

\section{EXAMPLE}

The following numerical example is used to compare the performances of the proposed approaches. The process to be identified is described by equation (1), where

$$
\begin{aligned}
G_{0}(q) & =\frac{0.5 q^{-1}}{1-0.8 q^{-1}}, n=1 \\
C(q) & =\frac{0.0012+0.0002 q^{-1}-0.001 q^{-2}}{0.5-0.9656 q^{-1}+0.4656 q^{-2}}, m=2 \\
H_{0}(q) & =\frac{1-1.56 q^{-1}+1.045 q^{-2}-0.3338 q^{-3}}{1-2.35 q^{-1}+2.09 q^{-2}-0.6675 q^{-3}}
\end{aligned}
$$

$r(t)$ is a deterministic sequence (realization of a random binary signal) and $e_{0}(t)$ is a white noise uncorrelated with $r(t)$. The process parameters are estimated by means of the methods M1 to M4. Moreover, the results from the basic closed-loop IV method developed by Söderström et al. (1987) are also analyzed. This methods referenced as M5, consists in using the delayed version of the reference signal as instruments; the estimate is thus given by

$$
\begin{aligned}
& \hat{\theta}_{\text {cliv }}=\left[\sum_{t=1}^{N} \zeta(t) \varphi^{T}(t)\right]^{-1}\left[\sum_{t=1}^{N} \zeta(t) y(t)\right] \\
& \zeta(t)=\left[\begin{array}{llll}
r(t) & r(t-1) & \cdots & r(t-2 n)
\end{array}\right]^{T}
\end{aligned}
$$

Summary of methods:

M1: tailor-made IV (tiv) / BELS with $m>n$, see section 3.3;

M2: bootstrap IV (cliv4), see section 5.1;

M3: bootstrap IV with automated noise model identification (cliv4-armasel), see section 5.1;

M4: bootstrap IV with high-order least-squares (cliv3), see section 5.2;

M5: basic closed-loop IV.

For illustration purposes, all of these methods are compared to a benchmark which consists in applying the true noise and process models for generating the prefilter and the instruments.

The process parameters are estimated on the basis of closed-loop data sequences of length $N=1000$. Monte Carlo simulation of 100 experiments has been performed for a signal to noise ratio

$$
S N R=10 \log \left(\frac{P_{y_{d}}}{P_{e}}\right)=15 \mathrm{~dB},
$$

where $P_{x}$ denotes the power of the signal $x$ and $y_{d}$ is the noise-free output signal.

In figure 2, the Bode diagrams of the 100 models identified by the six methods are represented. Furthermore, the following function is computed and represented in figure 3 for each algorithm

$$
g(\omega)=\frac{1}{M C} \sum_{k=1}^{M C}\left|G_{0}\left(e^{i \omega}\right)-\hat{G}_{k}\left(e^{i \omega}\right)\right|
$$

where $M C$ denotes the number of Monte Carlo experimentations and $\hat{G}_{k}\left(e^{i \omega}\right)$ the transfer function estimated during the $k^{\text {th }}$ Monte Carlo experimentation. 
Figures 2 and 3 show that M3 gives the best results (no bias, lower standard-deviation), really close to those of the benchmark. The two approximate versions of the optimal IV algorithm (M3, M4) and the closedloop IV method (M5) give better results than the proposed extended IV (M1) in that case. Moreover, the method based on the least-square high-order model (M4) seems to be more appropriate than the extension of the IV4 method to this closed-loop case (M2).

Furthermore, the 2 norm of the difference between the real and estimated transfer functions is also computed for each method

$$
N o r m=\frac{1}{M C} \sum_{k=1}^{M C} \int\left|G_{0}\left(e^{i \omega}\right)-\hat{G}_{k}\left(e^{i \omega}\right)\right|^{2} d \omega
$$

The results are given in table 1 and confirm the previous graphic results: the bootstrap methods considered in the paper give better results than the extended IV technique.

\begin{tabular}{|c|c|c|c|c|c|c|}
\hline method & bench. & $\begin{array}{r}\text { M1 } \\
\text { (tiv) }\end{array}$ & $\begin{array}{l}\text { M2 } \\
(\text { cliv4) }\end{array}$ & $\begin{array}{c}\text { M3 } \\
\text { (armasel) }\end{array}$ & $\begin{array}{l}\text { M4 } \\
(\operatorname{cliv3})\end{array}$ & $\begin{array}{l}\text { M5 } \\
\text { (cliv) }\end{array}$ \\
\hline Norm & 1.921 & 4.766 & 2.893 & 2.223 & 2.591 & 3.685 \\
\hline
\end{tabular}

Table 1. Norm

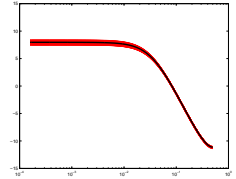

(a) benchmark

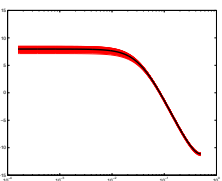

(d) M3:

cliv4-

armasel

Fig. 2. Bode amplitude plots of the process (black) and of the estimates (grey)

\section{CONCLUSION}

Several IV and IV-related estimators for closed-loop system identification have been studied and set in an extended IV framework. An explicit expression for the covariance matrix of estimation errors is given and it is then shown that a minimal value of this covariance matrix can be achieved for a particular choice of instruments and prefilters. This minimal value requires the knowledge of the true system parameters and is therefore not reachable in practice. Several methods have thus been developed to determine the design parameters which allow to approximate the optimal

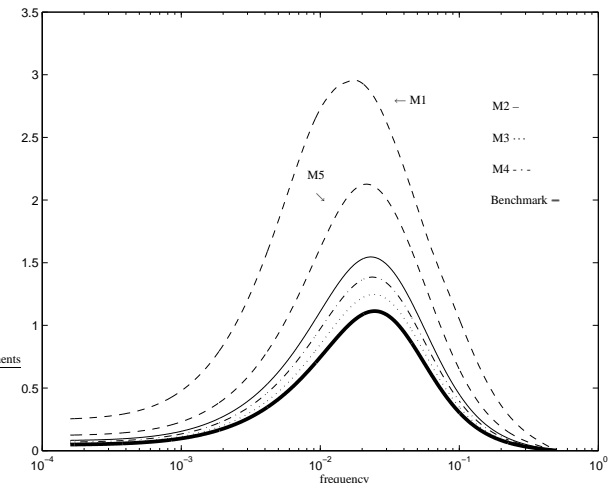

Fig. 3. Average frequency response error $g(\omega)$ for several IV methods; results are averaged over 100 Monte Carlo experiments.

closed-loop IV estimator. These methods have been compared to the recently suggested Tailor-made IV methods and BELS methods which are known to lead to unbiased plant estimates in closed loop. However for arriving at estimates with attractive variance properties it is preferably to apply bootstrap IV methods as considered in this paper.

\section{ACKNOWLEDGMENT}

The authors wish to thank A. Richard for useful discussions.

\section{APPENDIX}

Proof of Proposition 1. Using (15), the solution to (16) can be written as

$$
\hat{\theta}_{t i v}(N)=\left[\sum_{t=1}^{N} \eta(t) \bar{\varphi}^{T}(t)\right]^{-1}\left[\sum_{t=1}^{N} \eta(t) \bar{y}(t)\right] .
$$

With (20) the expression (17) for $F$ can be written as

$$
F=\hat{R}_{\varphi_{r} \bar{\varphi}}^{T}\left(\hat{R}_{\varphi_{r} \varphi_{c}} \hat{R}_{\varphi_{r} \varphi_{c}}^{T}\right)^{-1} .
$$

Substituting $\eta(t)=F \varphi_{r}(t)$ into the expression for $\hat{\theta}_{t i v}(N)$, and using (8) then delivers

$$
\begin{aligned}
\hat{\theta}_{t i v}=\left[\hat{R}_{\varphi_{r} \bar{\varphi}}^{T}\left(\hat{R}_{\varphi_{r} \varphi_{c}} \hat{R}_{\varphi_{r} \varphi_{c}}^{T}\right)^{-1} \hat{R}_{\varphi_{r} \bar{\varphi}}\right]^{-1} & \\
\cdot & \hat{R}_{\varphi_{r} \bar{\varphi}}^{T}\left(\hat{R}_{\varphi_{r} \varphi_{c}} \hat{R}_{\varphi_{r} \varphi_{c}}^{T}\right)^{-1} \hat{R}_{\varphi_{r} \bar{y}}
\end{aligned}
$$

The structure of this expression is

$$
\hat{\theta}_{\text {tiv }}=\left(A^{T} Q A\right)^{-1} A^{T} Q B
$$

with

$$
A=\hat{R}_{\varphi_{r} \bar{\varphi}}, Q=\left(\hat{R}_{\varphi_{r} \varphi_{c}} \hat{R}_{\varphi_{r} \varphi_{c}}^{T}\right)^{-1}, B=\hat{R}_{\varphi_{r} \bar{y}}
$$

having the structure of a solution to a weighted leastsquares problem. As a result, $\hat{\theta}_{t i v}$ is the solution to the extended IV problem

$$
\hat{\theta}_{t i v}=\arg \min _{\theta}\left\|\hat{R}_{\varphi_{r} \bar{\varphi}} \theta-\hat{R}_{\varphi_{r}}\right\|_{Q}^{2}
$$

with weighting matrix $Q$ given by equation (50). 


\section{REFERENCES}

Broersen, P.M.T. (2002). Automatic spectral analysis with time series models. IEEE Trans. Instrum. Measurem. 51(2), 211-216.

Forssell, U. and C.T. Chou (1998). Efficiency of prediction error and instrumental variable methods for closed-loop identification. In: 37th IEEE Conf. Decision and Control. Tampa - USA. pp. 1287-1288.

Forssell, U. and L. Ljung (1999). Closed-loop identification revisited. Automatica 35(7), 1215-1241.

Gilson, M. and P. Van den Hof (2001). On the relation between a bias-eliminated least-squares (BELS) and an IV estimator in closed-loop identification. Automatica 37(10), 1593-1600.

Ljung, L. (1999). System Identification : Theory for the User, Second Edition, Prentice-Hall.

Söderström, T. and P. Stoica (1983). Instrumental Variable Methods for System Identification. Springer-Verlag.

Söderström, T. and P. Stoica (1989). System Identification. Prentice-Hall.

Söderström, T., P. Stoica and E. Trulsson (1987). Instrumental variable methods for closed-loop systems. In: 10th IFAC World Congress on Automatic Control. Munich - Germany. pp. 363-368.

Söderström, T., W.X. Zheng and P. Stoica (1999). Comments on "on a least-squares-based algorithm for identification of stochastic linear systems". IEEE Trans. Signal Processing 47(5), 1395-1396.

Stoica, P. and T. Söderström (1983). Optimal instrumental variable estimation and approximate implementations. IEEE Trans. Automatic Control AC-28, 757-772.

Van den Hof, P.M.J. (1998). Closed-loop issues in system identification. Annual Reviews in Control 22, 173-186.

Young, P.C. (1976). Some observations on instrumental variable methods of time-series analysis. Int. J. Control 23(5), 593-612.

Zheng, W.X. (1996). Identification of closed-loop systems with low-order controllers. Automatica 32(12), 1753-1757.

Zheng, W.X. and C.B. Feng (1995). A bias-correction method for indirect identification of closed-loop systems. Automatica 31(7), 1019-1024. 